\title{
PROPERTIES OF MODIFIED RIEMANNIAN EXTENSIONS
}

\author{
AYDIN GEZER, LOKMAN BILEN, AND ALI CAKMAK
}

\begin{abstract}
Let $M$ be an $n$-dimensional differentiable manifold with a symmetric connection $\nabla$ and $T^{*} M$ be its cotangent bundle. In this paper, we study some properties of the modified Riemannian extension $\widetilde{g}_{\nabla, c}$ on $T^{*} M$ defined by means of a symmetric $(0,2)$-tensor field $c$ on $M$. We get the conditions under which $T^{*} M$ endowed with the horizontal lift ${ }^{H} J$ of an almost complex structure $J$ and with the metric $\widetilde{g}_{\nabla, c}$ is a Kähler-Norden manifold. Also curvature properties of the Levi-Civita connection and another metric connection of the metric $\widetilde{g}_{\nabla, c}$ are presented.
\end{abstract}

\section{Introduction}

Let $M$ be an $n$-dimensional differentiable manifold and $T^{*} M$ be its cotangent bundle. There is a well-known natural construction which yields, for any affine connection $\nabla$ on $M$, a pseudo-Riemannian metric $\widetilde{g}_{\nabla}$ on $T^{*} M$. The metric $\widetilde{g}_{\nabla}$ is called Riemannian extension of $\nabla$. Riemannian extensions were originally defined by Patterson and Walker [17] and further investigated in Afifi [2, thus relating pseudo-Riemannian properties of $T^{*} M$ with the affine structure of the base manifold $(M, \nabla)$. Moreover, Riemannian extensions were also considered in Garcia-Rio et al. 8] in relation to Osserman manifolds (see also Derdzinski [5]). Riemannian extensions provide a link between affine and pseudo-Riemannian geometries. Some properties of the affine connection $\nabla$ can be investigated by means of the corresponding properties of the Riemannian extension $\widetilde{g}_{\nabla}$. For instance, $\nabla$ is projectively flat if and only if $\widetilde{g}_{\nabla}$ is locally conformally flat [2]. For Riemannian extensions, also see 11, 7, 10, 12, 14, 18, 19, 21, 22. In [3, 4, the authors introduced a modification of the usual Riemannian extensions which is called modified Riemannian extension.

Almost complex Norden manifolds are among the most important geometrical structures which can be considered on a manifold. Let $M_{2 k}$ be a $2 k$-dimensional differentiable manifold endowed with an almost complex structure $J$ and a pseudoRiemannian metric $g$ of signature $(k, k)$ such that $g(J X, Y)=g(X, J Y)$, i.e. $g$ is pure with respect to $J$ for arbitrary vector fields $X$ and $Y$ on $M_{2 k}$. Then the metric $g$ is called Norden metric. Norden metrics are referred to as anti-Hermitian metrics or $B$-metrics. The study of such manifolds is interesting because there exists a difference between the geometry of a $2 k$-dimensional almost complex manifold with Hermitian metric and the geometry of a $2 k$-dimensional almost complex manifold with Norden metric. A notable difference between Norden metrics and Hermitian

2000 Mathematics Subject Classification. Primary 53C07, 53C55; Secondary 53C22.

Key words and phrases. Almost complex structure, cotangent bundle, geodesic, holomorphic tensor field, Riemannian extension, Riemannian curvature tensors. 
metrics is that $G(X, Y)=g(X, J Y)$ is another Norden metric, rather than a differential 2-form. Some authors considered almost complex Norden structures on the cotangent bundle [6, 15, 16.

In this paper we will use a deformation of the Riemannian extension on the cotangent bundle $T^{*} M$ over $(M, \nabla)$ by means of a symmetric tensor field $c$ on $M$, where $\nabla$ is a symmetric affine connection on $M$. The metric is so-called modified Riemannian extenson. In section 3 , in the particular case where $\nabla$ is the Levi-Civita connection on a Riemannian manifold $(M, g)$, we get the conditions under which the almost complex manifold with Norden metric $\left(T^{*} M,{ }^{H} J, \widetilde{g}_{\nabla, c}\right)$ is a Kähler-Norden manifold, where ${ }^{H} J$ is the horizontal lift of an almost complex structure $J$ and $\widetilde{g}_{\nabla, c}$ is the modified Riemannian extension. In section 4 and 5 , we show that the geometric properties of the Levi-Civita connection and another metric connection of the modified Riemannian extension $\widetilde{g}_{\nabla, c}$.

Throughout this paper, all manifolds, tensor fields and connections are always assumed to be differentiable of class $C^{\infty}$. Also, we denote by $\Im_{q}^{p}(M)$ the set of all tensor fields of type $(p, q)$ on $M$, and by $\Im_{q}^{p}\left(T^{*} M\right)$ the corresponding set on the cotangent bundle $T^{*} M$. The Einstein summation convention is used, the range of the indices $i, j, s$ being always $\{1,2, \ldots, n\}$

\section{Preliminaries}

2.1. The cotangent bundle. Let $M$ be an $n$-dimensional smooth manifold and denote by $\pi: T^{*} M \rightarrow M$ its cotangent bundle with fibres the cotangent spaces to $M$. Then $T^{*} M$ is a $2 n$-dimensional smooth manifold and some local charts induced naturally from local charts on $M$, may be used. Namely, a system of local coordinates $\left(U, x^{i}\right), i=1, \ldots, n$ in $M$ induces on $T^{*} M$ a system of local coordinates $\left(\pi^{-1}(U), x^{i}, x^{\bar{i}}=p_{i}\right), \bar{i}=n+i=n+1, \ldots, 2 n$, where $x^{\bar{i}}=p_{i}$ is the components of covectors $p$ in each cotangent space $T_{x}^{*} M, x \in U$ with respect to the natural coframe $\left\{d x^{i}\right\}$.

Let $X=X^{i} \frac{\partial}{\partial x^{i}}$ and $\omega=\omega_{i} d x^{i}$ be the local expressions in $U$ of a vector field $X$ and a covector (1-form) field $\omega$ on $M$, respectively. Then the vertical lift ${ }^{V} \omega$ of $\omega$, the horizontal lift ${ }^{H} X$ and the complete lift ${ }^{C} X$ of $X$ are given, with respect to the induced coordinates, by

$$
\begin{gathered}
{ }^{V} \omega=\omega_{i} \partial_{\bar{i}}, \\
{ }^{H} X=X^{i} \partial_{i}+p_{h} \Gamma_{i j}^{h} X^{j} \partial_{\bar{i}}
\end{gathered}
$$

and

$$
{ }^{C} X=X^{i} \partial_{i}-p_{h} \partial_{i} X^{h} \partial_{\bar{i}}
$$

where $\partial_{i}=\frac{\partial}{\partial x^{2}}, \partial_{\bar{i}}=\frac{\partial}{\partial x^{i}}$ and $\Gamma_{i j}^{h}$ are the coefficients of a symmetric (torsion-free) affine connection $\nabla$ in $M$.

The Lie bracket operation of vertical and horizontal vector fields on $T^{*} M$ is given by the formulas

$$
\left\{\begin{array}{l}
{\left[{ }^{H} X,{ }^{H} Y\right]={ }^{H}[X, Y]+{ }^{V}(p \circ R(X, Y))} \\
\left.{ }^{H} X, V \omega\right]={ }^{V}\left(\nabla_{X} \omega\right) \\
\left.{ }^{V} \theta,{ }^{V} \omega\right]=0
\end{array}\right.
$$


for any $X, Y \Im_{0}^{1}(M)$ and $\theta, \omega \in \Im_{1}^{0}(M)$, where $R$ is the curvature tensor of the symmetric connection $\nabla$ defined by $R(X, Y)=\left[\nabla_{X}, \nabla_{Y}\right]-\nabla_{[X, Y]}$ (for details, see [24]).

2.2. Expressions in the adapted frame. We insert the adapted frame which allows the tensor calculus to be efficiently done in $T^{*} M$. With the symmetric affine connection $\nabla$ in $M$, we can introduce adapted frames on each induced coordinate neighborhood $\pi^{-1}(U)$ of $T^{*} M$. In each local chart $U \subset M$, we write $X_{(j)}=\frac{\partial}{\partial x^{j}}$, $\theta^{(j)}=d x^{j}, j=1, \ldots, n$. Then from (2.1) and (2.2), we see that these vector fields have, respectively, local expressions

$$
\begin{gathered}
{ }^{H} X_{(j)}=\partial_{j}+p_{a} \Gamma_{h j}^{a} \partial_{\bar{h}} \\
{ }^{V} \theta^{(j)}=\partial_{\bar{j}}
\end{gathered}
$$

with respect to the natural frame $\left\{\partial_{j}, \partial_{j}\right\}$. These $2 n$ vector fields are linearly independent and they generate the horizontal distribution of $\nabla$ and the vertical distribution of $T^{*} M$, respectively. The set $\left\{{ }^{H} X_{(j)},{ }^{V} \theta^{(j)}\right\}$ is called the frame adapted to the connection $\nabla$ in $\pi^{-1}(U) \subset T^{*} M$. By denoting

$$
\begin{aligned}
& E_{j}={ }^{H} X_{(j)}, \\
& E_{\bar{j}}={ }^{V} \theta^{(j)},
\end{aligned}
$$

we can write the adapted frame as $\left\{E_{\alpha}\right\}=\left\{E_{j}, E_{\bar{j}}\right\}$. The indices $\alpha, \beta, \gamma, \ldots=$ $1, \ldots, 2 n$ indicate the indices with respect to the adapted frame.

Using (2.1), (2.2) and (2.4), we have

$$
V_{\omega}=\left(\begin{array}{l}
0 \\
\omega_{j}
\end{array}\right)
$$

and

$$
{ }^{H} X=\left(\begin{array}{l}
X^{j} \\
0
\end{array}\right)
$$

with respect to the adapted frame $\left\{E_{\alpha}\right\}$ (for details, see 24]). By the straightforward calculations, we have the lemma below.

Lemma 1. The Lie brackets of the adapted frame of $T^{*} M$ satisfy the following identities:

$$
\begin{aligned}
{\left[E_{i}, E_{j}\right] } & =p_{s} R_{i j l}^{s} E_{\bar{l}}, \\
{\left[E_{i}, E_{\bar{j}}\right] } & =-\Gamma_{i l}^{j} E_{\bar{l}}, \\
{\left[E_{\bar{i}}, E_{\bar{j}}\right] } & =0
\end{aligned}
$$

where $R_{i j l}{ }^{s}$ denote the components of the curvature tensor of the symmetric connection $\nabla$ on $M$. 


\section{KÄHLER-NORDEN STRUCTURES ON THE COTANGENT BUNDLE}

An almost complex Norden manifold $(M, J, g)$ (an almost complex manifold with a Norden metric) is defined to be a real $2 n$-dimensional differentiable manifold $M$, i.e. $J$ is an almost complex structure and $g$ is a pseudo-Riemannian metric of neutral signature $(n, n)$ on $M$ such that:

$$
J^{2} X=-X, g(J X, Y)=g(X, J Y)
$$

for all $X, Y \in \Im_{0}^{1}(M)$. An Kähler-Norden (anti-Kähler) manifold can be defined as a triple $(M, J, g)$ which consists of a smooth manifold $M$ endowed with an almost complex structure $J$ and a Norden metric $g$ such that $\nabla J=0$, where $\nabla$ is the LeviCivita connection of $g$. It is well known that the condition $\nabla J=0$ is equivalent to C-holomorphicity (analyticity) of the Norden metric $g$ [11], i.e. $\Phi_{J} g=0$, where $\Phi_{J}$ is the Tachibana operator [23, 20]: $\left(\Phi_{J} g\right)(X, Y, Z)=(J X)(g(Y, Z))-X(g(J Y, Z))+$ $g\left(\left(L_{Y} J\right) X, Z\right)+g\left(Y,\left(L_{Z} J\right) X\right)$. Also note that $G(Y, Z)=(g \circ J)(Y, Z)=g(J Y, Z)$ is the twin Norden metric. Since in dimension 2 an Kähler-Norden manifold is flat, we assume in the sequel that $\operatorname{dim} M \geq 4$.

Next, for a given symmetric connection $\nabla$ on $M$, the cotangent bundle $T^{*} M$ may be equipped with a pseudo-Riemannian metric $\widetilde{g}_{\nabla}$ of signature $(n, n)$ : the Riemannian extension of $\nabla$ [17, given by

$$
\widetilde{g}_{\nabla}\left({ }^{C} X,{ }^{C} Y\right)=-\gamma\left(\nabla_{X} Y+\nabla_{Y} X\right)
$$

where ${ }^{C} X,{ }^{C} Y$ denote the complete lifts to $T^{*} M$ of vector fields $X, Y$ on $M$. Moreover for any vector field $Z$ on $M, Z=Z^{i} \partial_{i}, \gamma Z$ is the function on $T^{*} M$ defined by $\gamma Z=p_{i} Z^{i}$. The Riemannian extension is expressed by

$$
\widetilde{g}_{\nabla}=\left(\begin{array}{cc}
-2 p_{h} \Gamma_{i j}^{h} & \delta_{j}^{i} \\
\delta_{i}^{j} & 0
\end{array}\right)
$$

with respect to the natural frame.

Now we give a deformation of the Riemannian extension above by means of a symmetric $(0,2)$-tensor field $c$ on $M$, which the metric is called modified Riemannian extension. The modified Riemannian extension is expressed by

$$
\widetilde{g}_{\nabla, c}=g_{\nabla}+\pi^{*} c=\left(\begin{array}{cc}
-2 p_{h} \Gamma_{i j}^{h}+c_{i j} & \delta_{j}^{i} \\
\delta_{i}^{j} & 0
\end{array}\right) .
$$

with respect to the natural frame. It follows that the signature of $\widetilde{g}_{\nabla, c}$ is $(n, n)$.

Denote by $\nabla$ the Levi-Civita connection of a pseudo-Riemannian metric $g$. In the section, we will consider $T^{*} M$ equipped with the metric $\widetilde{g}_{\nabla, c}$ over a pseudoRiemannian manifold $(M, g)$. Since the vector fields ${ }^{H} X$ and ${ }^{V} \omega$ span the module of vector fields on $T^{*} M$, any tensor field is determined on $T^{*} M$ by their actions on ${ }^{H} X$ and ${ }^{V} \omega$. The modified Riemannian extension $\widetilde{g}_{\nabla, c}$ has the following properties

$$
\begin{aligned}
\widetilde{g}_{\nabla, c}\left({ }^{H} X,{ }^{H} Y\right) & =c(X, Y) \\
\widetilde{g}_{\nabla, c}\left({ }^{H} X,{ }^{V} \omega\right) & =g_{\nabla, c}\left({ }^{V} \omega,{ }^{H} X\right)=\omega(X) \\
\widetilde{g}_{\nabla, c}\left({ }^{V} \omega,{ }^{V} \theta\right) & =0
\end{aligned}
$$

for all $X, Y \in \Im_{0}^{1}(M)$ and $\omega, \theta \in \Im_{1}^{0}(M)$, which characterize $\widetilde{g}_{\nabla, c}$. 
The horizontal lift of a $(1,1)$-tensor field $J$ to $T^{*} M$ is defined by:

$$
\begin{aligned}
{ }^{H} J\left({ }^{H} X\right) & ={ }^{H}(J X) \\
{ }^{H} J\left({ }^{V} \omega\right) & ={ }^{V}(\omega \circ J)
\end{aligned}
$$

for any $X \in \Im_{0}^{1}(M)$ and $\omega \in \Im_{1}^{0}(M)$. Moreover, it is well known that if $J$ is an almost complex structure on $(M, g)$, then its horizontal lift ${ }^{H} J$ is an almost complex structure on $T^{*} M[24$.

Putting

$$
A(\tilde{X}, \tilde{Y})=\widetilde{g}_{\nabla, c}\left({ }^{H} J \widetilde{X}, \widetilde{Y}\right)-\widetilde{g}_{\nabla, c}\left(\widetilde{X},{ }^{H} J \widetilde{Y}\right)
$$

for any $\tilde{X}, \widetilde{Y} \in \Im_{0}^{1}\left(T^{*} M\right)$. For all vector fields $\widetilde{X}$ and $\tilde{Y}$ which are of the form ${ }^{V} \omega,{ }^{V} \theta$ or ${ }^{H} X,{ }^{H} Y$, from (3.2) and (3.3), we have

$$
\begin{aligned}
A\left({ }^{H} X,{ }^{H} Y\right) & =\widetilde{g}_{\nabla, c}\left({ }^{H} J\left({ }^{H} X\right),{ }^{H} Y\right)-\widetilde{g}_{\nabla, c}\left({ }^{H} X,{ }^{H} J\left({ }^{H} Y\right)\right) \\
& =\widetilde{g}_{\nabla, c}\left({ }^{H}(J X),{ }^{H} Y\right)-\widetilde{g}_{\nabla, c}\left({ }^{H} X,{ }^{H}(J Y)\right) \\
& =c(J X, Y)-c(X, J Y) \\
A\left({ }^{H} X,{ }^{V} \theta\right) & =\widetilde{g}_{\nabla, c}\left({ }^{H} J\left({ }^{H} X\right),{ }^{V} \theta\right)-\widetilde{g}_{\nabla, c}\left({ }^{H} X,{ }^{H} J\left({ }^{V} \theta\right)\right) \\
& =\widetilde{g}_{\nabla, c}\left({ }^{H}(J X),{ }^{V} \theta\right)-\widetilde{g}_{\nabla, c}\left({ }^{H} X,{ }^{V}(\theta \circ J)\right) \\
& =\theta(J X)-(\theta \circ J)(X) \\
A\left({ }^{V} \omega,{ }^{H} Y\right) & =\widetilde{g}_{\nabla, c}\left({ }^{H} J\left({ }^{V} \omega\right),{ }^{H} Y\right)-\widetilde{g}_{\nabla, c}\left({ }^{V} \omega,{ }^{H} J\left({ }^{H} X\right)\right) \\
& =(\omega \circ J)(Y)-\omega(J X) \\
A\left({ }^{V} \omega,{ }^{V} \theta\right) & =\widetilde{g}_{\nabla, c}\left({ }^{H} J\left({ }^{V} \omega\right),{ }^{V} \theta\right)-\widetilde{g}_{\nabla, c}\left({ }^{V} \omega,{ }^{H} J\left({ }^{V} \theta\right)\right) \\
& =\widetilde{g}_{\nabla, c}\left({ }^{H} J\left({ }^{V} \omega\right),{ }^{V} \theta\right)-\widetilde{g}_{\nabla, c}\left({ }^{V} \omega,{ }^{H} J\left({ }^{V} \theta\right)\right) \\
& =\widetilde{g}_{\nabla, c}\left({ }^{V}(\omega \circ J),{ }^{V} \theta\right)-\widetilde{g}_{\nabla, c}\left({ }^{V} \omega,{ }^{V}(\theta \circ J)\right) \\
& =0 .
\end{aligned}
$$

If $g$ and $c$ is pure with respect to $J$, we say that $A(\tilde{X}, \tilde{Y})=0$, i.e. $g_{\nabla, c}$ is pure with respect to ${ }^{H} \mathrm{~J}$.

We now are interested in the holomorphy property of the metric $g_{\nabla, c}$ with respect to ${ }^{H} J$. We calculate

$$
\begin{aligned}
\left(\Phi_{H}{ }_{J} \widetilde{g}_{\nabla, c}\right)(\tilde{X}, \tilde{Y}, \tilde{Z}) & =\left({ }^{H} J \tilde{X}\right)\left(\widetilde{g}_{\nabla, c}(\tilde{Y}, \tilde{Z})\right)-\tilde{X}\left(\widetilde{g}_{\nabla, c}\left({ }^{H} J \tilde{Y}, \tilde{Z}\right)\right) \\
& +\widetilde{g}_{\nabla, c}\left(\left(L_{\tilde{Y}}{ }^{H} J\right) \tilde{X}, \tilde{Z}\right)+\widetilde{g}_{\nabla, c}\left(\tilde{Y},\left(L_{\tilde{Z}}{ }^{H} J\right) \tilde{X}\right)
\end{aligned}
$$


for all $\tilde{X}, \tilde{Y}, \tilde{Z} \in \Im_{0}^{1}\left(T^{*} M\right)$. Then we obtain the following equations

$$
\begin{aligned}
& \left(\Phi_{H} J \widetilde{g}_{\nabla, c}\right)\left({ }^{V} \omega,{ }^{V} \theta,{ }^{H} Z\right)=0, \\
& \left(\Phi_{H} J \widetilde{g}_{\nabla, c}\right)\left({ }^{V} \omega,{ }^{V} \theta,{ }^{V} \sigma\right)=0, \\
& \left(\Phi_{H} J \widetilde{g}_{\nabla, c}\right)\left({ }^{V} \omega,{ }^{H} Y,{ }^{V} \sigma\right)=0, \\
& \left(\Phi_{H} J \widetilde{g}_{\nabla, c}\right)\left({ }^{V} \omega,{ }^{H} Y,{ }^{H} Z\right)=\left(\omega \circ \nabla_{Y} J\right)(Z)+\left(\omega \circ \nabla_{Z} J\right)(Y), \\
& \left(\Phi_{H} \widetilde{g}_{\nabla, c}\right)\left({ }^{H} X,{ }^{V} \omega,{ }^{H} Z\right)=\left(\Phi_{J} g\right)(X, \widetilde{\omega}, Z)-g\left(\left(\nabla_{\widetilde{\omega}} J\right) X, Z\right), \\
& \left(\Phi_{H} J \widetilde{g}_{\nabla, c}\right)\left({ }^{H} X,{ }^{V} \omega,{ }^{V} \sigma\right)=0, \\
& \left.\left(\Phi_{H} \widetilde{g}_{\nabla, c}\right)\left({ }^{H} X,{ }^{H} Y,{ }^{H} Z\right)=\left(\Phi_{J} c\right)(X, Y, Z)\right) \\
& +(p \circ R(Y, J X)-p \circ R(Y, X) J)(Z) \\
& +(p \circ R(Z, J X)-p \circ R(Z, X) J)(Y), \\
& \left(\Phi_{H} \widetilde{g}_{\nabla, c}\right)\left({ }^{H} X,{ }^{H} Y,{ }^{V} \sigma\right)=\left(\Phi_{J} g\right)(X, Y, \widetilde{\sigma})-g\left(Y,\left(\nabla_{\widetilde{\sigma}} J\right) X\right),
\end{aligned}
$$

where $\widetilde{\omega}=g^{-1} \circ \omega=g^{i j} \omega_{j}$ is the associated vector field of $\omega$. On the other hand, we know that the Riemannian curvature $R$ of Kähler-Norden manifolds is totally pure. Also, the condition $\Phi_{J} g=0$ is equivalent to $\nabla J=0$, where $\nabla$ is the Levi-Civita connection of $g$. Hence, we say following result.

Theorem 1. Let $(M, J, g)$ is a Kähler-Norden manifold. Then $T^{*} M$ is a KählerNorden manifold equipped with the metric $\widetilde{g}_{\nabla, c}$ and the almost complex structure ${ }^{H} J$ if and only if the symmetric $(0,2)$-tensor field $c$ on $M$ is a holomorphic tensor field with respect to the almost complex structure $J$.

\section{Curvature properties of the Levi-Civita connection of the MODIFIED RIEMANNIAN EXTENSION $\widetilde{g}_{\nabla, c}$}

From now on we will consider $T^{*} M$ equipped with the modified Riemannian extension $\widetilde{g}_{\nabla, c}$ for a given symmetric connection $\nabla$ on $M$. By virtue of (2.5) and (2.6), the modified Riemannian extension $\widetilde{g}_{\nabla, c}$ and its inverse $\bar{g}_{\nabla, c}$ have the following components with respect to the adapted frame $\left\{E_{\alpha}\right\}$ :

$$
\begin{aligned}
\left(\widetilde{g}_{\nabla, c}\right)_{\beta \gamma} & =\left(\begin{array}{cc}
c_{i j} & \delta_{i}^{j} \\
\delta_{j}^{i} & 0
\end{array}\right) . \\
\left(\bar{g}_{\nabla, c}\right)^{\beta \gamma} & =\left(\begin{array}{cc}
0 & \delta_{j}^{i} \\
\delta_{i}^{j} & -c_{i j}
\end{array}\right) .
\end{aligned}
$$

The Levi-Civita connection $\widetilde{\nabla}$ of $\widetilde{g}_{\nabla, c}$ is characterized by the Koszul formula:

$$
\begin{aligned}
& 2 \widetilde{g}_{\nabla, c}\left(\widetilde{\nabla} \widetilde{X}_{\tilde{Y}}, \widetilde{Z}\right)=\tilde{X}\left(\widetilde{g}_{\nabla, c}(\tilde{Y}, \widetilde{Z})\right)+\widetilde{Y}\left(\widetilde{g}_{\nabla, c}(\widetilde{Z}, \widetilde{X})\right)-\widetilde{Z}\left(\widetilde{g}_{\nabla, c}(\widetilde{X}, \widetilde{Y})\right) \\
& -\widetilde{g}_{\nabla, c}(\tilde{X},[\tilde{Y}, \widetilde{Z}])+\widetilde{g}_{\nabla, c}(\tilde{Y},[\widetilde{Z}, \widetilde{X}])+\widetilde{g}_{\nabla, c}(\widetilde{Z},[\tilde{X}, \widetilde{Y}])
\end{aligned}
$$

for all vector fields $\tilde{X}, \widetilde{Y}$ and $\widetilde{Z}$ on $T^{*} M$. One can verify the Koszul formula for pairs $\widetilde{X}=E_{i}, E_{\bar{i}}$ and $\widetilde{Y}=E_{j}, E_{\bar{j}}$ and $\widetilde{Z}=E_{k}, E_{\bar{k}}$. By using (4.1), Lemma 1 we obtain the following result.

Proposition 1. The Levi-Civita connection $\widetilde{\nabla}$ of the modified Riemannian exten- 
sion $\widetilde{g}_{\nabla, c}$ is given by

$$
\begin{aligned}
& \widetilde{\nabla}_{E_{\bar{i}}} E_{\bar{j}}=0, \widetilde{\nabla}_{E_{\bar{i}}} E_{j}=0 \\
& \widetilde{\nabla}_{E_{i}} E_{\bar{j}}=-\Gamma_{i h}^{j} E_{\bar{h}} \\
& \widetilde{\nabla}_{E_{i}} E_{j}=\Gamma_{i j}^{h} E_{h}+\left\{p_{s} R_{h j i}{ }^{s}+\frac{1}{2}\left(\nabla_{i} c_{j h}+\nabla_{j} c_{i h}-\nabla_{h} c_{i j}\right)\right\} E_{\bar{h}}
\end{aligned}
$$

where $R_{h j i}{ }^{s}$ are the local coordinate components of the curvature tensor field $R$ of the symmetric connection $\nabla$ on $M$.

An important geometric problem is to find the geodesics on the smooth manifolds with respect to the Riemannian metrics. Let $C$ be a curve in $M$ expressed locally by $x^{h}=x^{h}(t)$. We define a curve $\widetilde{C}$ in $T^{*} M$ by

$$
\left\{\begin{array}{l}
x^{h}=x^{h}(t) \\
x^{\bar{h}} \stackrel{\text { def }}{=} p_{h}=\omega_{h}(t)
\end{array}\right.
$$

where $\omega_{h}(t)$ is a covector field along $C$. The geodesics of the connection $\widetilde{\nabla}$ is given by the differential equations

$$
\frac{\delta^{2} x^{A}}{d t^{2}}=\frac{d^{2} x^{A}}{d t^{2}}+\widetilde{\Gamma}_{C B}^{A} \frac{d x^{C}}{d t} \frac{d x^{B}}{d t}=0
$$

with respect to the induced coordinates $\left(x^{h}, x^{\bar{h}}\right)$, where $t$ is the arc length of a curve in $T^{*} M$.

We write down the form equivalent to (4.4), namely,

$$
\frac{d}{d t}\left(\frac{\theta^{\alpha}}{d t}\right)+\widetilde{\Gamma}_{\gamma \beta}^{\alpha} \frac{\theta^{\gamma}}{d t} \frac{\theta^{\beta}}{d t}=0
$$

with respect to adapted frame $\left\{E_{\alpha}\right\}$, where

$$
\begin{aligned}
& \frac{\theta^{h}}{d t}=\frac{d x^{h}}{d t}, \\
& \frac{\theta^{\bar{h}}}{d t}=\frac{\delta p_{h}}{d t}
\end{aligned}
$$

along a curve $x^{A}=x^{A}(t)$ in $T^{*} M$ [24]. Taking account of Proposition 1] then we have

$$
\left\{\begin{array}{l}
\text { (a) } \frac{d^{2} x^{h}}{d t^{2}}+\Gamma_{i j}^{h} \frac{d x^{i}}{d t} \frac{d x^{j}}{d t}=0, \\
\text { (b) } \frac{\delta^{2} p_{h}}{d t^{2}}+\left\{p_{s} R_{h j i}{ }^{s}+\frac{1}{2}\left(\nabla_{i} c_{j h}+\nabla_{j} c_{i h}-\nabla_{h} c_{i j}\right)\right\} \frac{d x^{i}}{d t} \frac{d x^{j}}{d t}=0
\end{array}\right.
$$

where $\frac{\delta^{2} p_{h}}{d t^{2}}=\frac{d}{d t}\left(\frac{\delta p_{h}}{d t}\right)-\Gamma_{i h}^{a} \frac{\delta p_{a}}{d t} \frac{d x^{i}}{d t}$. Thus we have the following result.

Theorem 2. Let $\tilde{C}$ be a curve on $T^{*} M$ and locally expressed by $x^{h}=x^{h}(t)$, $x^{\bar{h}}=p_{h}(t)$ with respect to the induced coordinates $\left(x^{h}, x^{\bar{h}}\right)$ in $\pi^{-1}(U) \subset T^{*} M$. The curve $\tilde{C}$ is a geodesic of the modified Riemannian extension $\widetilde{g}_{\nabla, c}$, if the projection $C$ of $\widetilde{C}$ is a geodesic in $M$ with the symmetric connection $\nabla$ and $p_{h}(t)$ satisfies the differential equation (b) in (4.5). 
The Riemannian curvature tensor $\widetilde{R}$ of $T^{*} M$ with the modified Riemannian extension $\widetilde{g}_{\nabla, c}$ is obtained from the well-known formula

$$
\widetilde{R}(\widetilde{X}, \widetilde{Y}) \widetilde{Z}=\widetilde{\nabla}_{\widetilde{X}} \widetilde{\nabla}_{\widetilde{Y}} \widetilde{Z}-\widetilde{\nabla}_{\widetilde{Y}} \widetilde{\nabla}_{\widetilde{X}} \widetilde{Z}-\widetilde{\nabla}_{[\widetilde{X}, \widetilde{Y}]} \widetilde{Z}
$$

for all $\widetilde{X}, \widetilde{Y}, \widetilde{Z} \in \Im_{0}^{1}\left(T^{*} M\right)$. Then from Lemma 1 and Proposition 1 , we get the following proposition.

Proposition 2. The components of the curvature tensor $\widetilde{R}$ of the cotangent bundle $T^{*} M$ with the modified Riemannian extension $\widetilde{g}_{\nabla, c}$ are given as follows:

$$
\begin{aligned}
\widetilde{R}\left(E_{i}, E_{j}\right) E_{k}= & R_{i j k}{ }^{h} E_{h} \\
& +\left\{p_{s}\left(\nabla_{i} R_{h k j}{ }^{s}-\nabla_{j} R_{h k i}{ }^{s}\right)\right. \\
& +\frac{1}{2}\left\{\nabla_{i}\left(\nabla_{k} c_{j h}-\nabla_{h} c_{j k}\right)-\nabla_{j}\left(\nabla_{k} c_{i h}-\nabla_{h} c_{i k}\right)\right. \\
& \left.\left.-R_{i j k}{ }^{m} c_{m h}-R_{i j h}{ }^{m} c_{k m}\right\}\right\} E_{\bar{h}} \\
\widetilde{R}\left(E_{i}, E_{j}\right) E_{\bar{k}}= & R_{j i h}{ }^{k} E_{\bar{h}} \\
\widetilde{R}\left(E_{i}, E_{\bar{j}}\right) E_{k}= & -R_{h k i}{ }^{j} E_{\bar{h}} \\
\widetilde{R}\left(E_{\bar{i}}, E_{j}\right) E_{k}= & R_{h k j}{ }^{i} E_{\bar{h}} \\
\widetilde{R}\left(E_{\bar{i}}, E_{j}\right) E_{\bar{k}}= & 0, \widetilde{R}\left(E_{i}, E_{\bar{j}}\right) E_{\bar{k}}=0 \\
\widetilde{R}\left(E_{\bar{i}}, E_{\bar{j}}\right) E_{k}= & 0, \widetilde{R}\left(E_{\bar{i}}, E_{\bar{j}}\right) E_{\bar{k}}=0
\end{aligned}
$$

with respect to the adapted frame $\left\{E_{\alpha}\right\}$.

Proposition 2 leads to the following result.

Theorem 3. Let $\nabla$ be a symmetric connection on $M$ and $T^{*} M$ be the cotangent bundle with the modified Riemannian extension $\widetilde{g}_{\nabla, c}$ over $(M, \nabla)$. Then $\left(T^{*} M, \widetilde{g}_{\nabla, c}\right)$ is locally flat if and only if $(M, \nabla)$ is locally flat and the components $c_{i j}$ of $c$ satisfy the condition

$$
\nabla_{i}\left(\nabla_{k} c_{j h}-\nabla_{h} c_{j k}\right)-\nabla_{j}\left(\nabla_{k} c_{i h}-\nabla_{h} c_{i k}\right)=0 .
$$

Let $\widetilde{X}$ and $\widetilde{Y}$ be vector fields of $T^{*} M$. The curvature operator $\widetilde{R}(\widetilde{X}, \widetilde{Y})$ is a differential operator on $T^{*} M$. Similarly, for vector fields $X$ and $Y$ of $M, R(X, Y)$ is a differential operator on $M$. Now, we operate the curvature operator to the curvature tensor. That is, for all $\widetilde{Z}, \widetilde{W}$ and $\widetilde{U}$, we consider the condition $(\widetilde{R}(\widetilde{X}, \widetilde{Y}) \widetilde{R})(\widetilde{Z}, \widetilde{W}) \widetilde{U}=$ 0.

The tensor $(\widetilde{R}(\widetilde{X}, \widetilde{Y}) \widetilde{R})(\widetilde{Z}, \widetilde{W}) \widetilde{U}$ has components

$$
\begin{aligned}
& ((\widetilde{R}(\widetilde{X}, \widetilde{Y}) \widetilde{R})(\widetilde{Z}, \widetilde{W}) \widetilde{U})_{\alpha \beta \gamma \theta \sigma}{ }^{\varepsilon} \\
= & \widetilde{R}_{\alpha \beta \tau} \widetilde{R}_{\gamma \theta \sigma} \widetilde{R}^{\tau}-\widetilde{R}_{\alpha \beta \gamma} \widetilde{R}_{\tau \theta \sigma}^{\varepsilon}-\widetilde{R}_{\alpha \beta \theta} \widetilde{R}_{\gamma \tau \sigma}^{\varepsilon}-\widetilde{R}_{\alpha \beta \sigma} \widetilde{R}_{\gamma \theta \tau} \widetilde{R}^{\varepsilon},
\end{aligned}
$$

with respect to the adapted frame $\left\{E_{\alpha}\right\}$. Similarly, for all $X, Y, Z, W, U$ on $M$,

$$
\begin{aligned}
& ((R(X, Y) R)(Z, W) U)_{i j k l m}{ }^{n} \\
= & R_{i j p}{ }^{n} R_{k l m}{ }^{p}-R_{i j k}{ }^{p} R_{p l m}{ }^{n}-R_{i j l}{ }^{p} R_{k p m}{ }^{n}-R_{i j m}{ }^{p} R_{k l p}{ }^{n} \\
= & 2 \nabla_{[i} \nabla_{j]} R_{k l m}{ }^{n}
\end{aligned}
$$

where $2 \nabla_{[i} \nabla_{j]}=\nabla_{i} \nabla_{j}-\nabla_{j} \nabla_{i}$. 
Case of $\alpha=i, \beta=j, \gamma=k, \theta=l, \sigma=\bar{m}, \varepsilon=\bar{n}$ in (4.8), by virtue of (4.6) the equation (4.8) reduces to

$$
\begin{aligned}
& ((\widetilde{R}(\widetilde{X}, \widetilde{Y}) \widetilde{R})(\widetilde{Z}, \widetilde{W}) \widetilde{U})_{i j k l \bar{m}}{ }^{\bar{n}} \\
= & \widetilde{R}_{i j p}{ }^{n} \widetilde{R}_{k l \bar{m}}{ }^{p}+\widetilde{R}_{i j \bar{p}}{ }^{n} \widetilde{R}_{k l \bar{m}}{ }^{\bar{p}}-\widetilde{R}_{i j k}{ }^{p} \widetilde{R}_{p l \bar{m}}{ }^{n}-\widetilde{R}_{i j k}{ }^{p} \widetilde{R}_{\bar{p} l \bar{m}}{ }^{n} \\
& -\widetilde{R}_{i j l}{ }^{p} \widetilde{R}_{k p \bar{m}}{ }^{n}-\widetilde{R}_{i j l}{ }^{p} \widetilde{R}_{k \overline{p m}}{ }^{n}-\widetilde{R}_{i j \bar{m}}{ }^{p} \widetilde{R}_{k l p}{ }^{n}-\widetilde{R}_{i j \bar{m}}{ }^{p} \widetilde{R}_{k l \bar{p}}{ }^{n} \\
= & -\left(R_{i j p}{ }^{m} R_{k l n}{ }^{p}-R_{i j k}{ }^{p} R_{p l n}{ }^{m}-R_{i j l}{ }^{p} R_{k p n}{ }^{m}-R_{i j n}{ }^{p} R_{k l p}{ }^{m}\right. \\
= & -2 \nabla_{[i} \nabla_{j]} R_{k l n}{ }^{m} \\
= & -((R(X, Y) R)(Z, W) U)_{i j k l n}{ }^{m} .
\end{aligned}
$$

Case of $\alpha=i, \beta=j, \gamma=\bar{k}, \theta=l, \sigma=m, \varepsilon=\bar{n}$ in (4.8), by virtue of (4.6), we have

$$
\begin{aligned}
& ((\widetilde{R}(\tilde{X}, \widetilde{Y}) \widetilde{R})(\widetilde{Z}, \widetilde{W}) \widetilde{U})_{i j \bar{k} l m}{ }^{n} \\
= & \widetilde{R}_{i j p}{ }^{n} \widetilde{R}_{\bar{k} l m}{ }^{p}+\widetilde{R}_{i j \bar{p}}{ }^{n} \widetilde{R}_{\bar{k} l m}{ }^{\bar{p}}-\widetilde{R}_{i j \bar{k}}{ }^{p} \widetilde{R}_{p l m}{ }^{n}-\widetilde{R}_{i j \bar{k}}{ }^{\bar{p}} \widetilde{R}_{\bar{p} l m}{ }^{\bar{n}} \\
& -\widetilde{R}_{i j l}{ }^{p} \widetilde{R}_{\bar{k} p m}{ }^{n}-\widetilde{R}_{i j l}{ }^{p} \widetilde{R}_{\bar{k} \bar{p} m}{ }^{n}-\widetilde{R}_{i j m}{ }^{p} \widetilde{R}_{\bar{k} l p}{ }^{n}-\widetilde{R}_{i j m}{ }^{\bar{p}} \widetilde{R}_{\bar{k} l \bar{p}}{ }^{n} \\
= & -\left(R_{i j p}{ }^{k} R_{n l m}{ }^{p}-R_{i j n}{ }^{p} R_{p l m}{ }^{k}-R_{i j l}{ }^{p} R_{n p m}{ }^{k}-R_{i j m}{ }^{p} R_{n l p}{ }^{k}\right. \\
= & -2 \nabla_{[i} \nabla_{j]} R_{n l m}{ }^{k} \\
= & -((R(X, Y) R)(Z, W) U)_{i j n l m}{ }^{k} .
\end{aligned}
$$

Case of $\alpha=i, \beta=j, \gamma=\bar{k}, \theta=\bar{l}, \sigma=\bar{m}, \varepsilon=\bar{n}$ in (4.8), by virtue of (4.6), it follows that

$$
((\widetilde{R}(\widetilde{X}, \widetilde{Y}) \widetilde{R})(\widetilde{Z}, \widetilde{W}) \widetilde{U})_{i j \overline{k l} \bar{m}}{ }^{\bar{n}}=0 .
$$

Case of $\alpha=i, \beta=j, \gamma=k, \theta=l, \sigma=m, \varepsilon=\bar{n}$ in (4.8), we obtain

$$
\begin{aligned}
& ((\widetilde{R}(\widetilde{X}, \widetilde{Y}) \widetilde{R})(\widetilde{Z}, \widetilde{W}) \widetilde{U})_{i j k l m}{ }^{n} \\
= & \widetilde{R}_{i j p}{ }^{n} \widetilde{R}_{k l m}{ }^{p}+\widetilde{R}_{i j \bar{p}}{ }^{\bar{n}} \widetilde{R}_{k l m}{ }^{p}-\widetilde{R}_{i j k}{ }^{p} \widetilde{R}_{p l m}{ }^{\bar{n}}-\widetilde{R}_{i j k}{ }^{\bar{p}} \widetilde{R}_{\bar{p} l m}{ }^{\bar{n}} \\
& -\widetilde{R}_{i j l}{ }^{p} \widetilde{R}_{k p m}{ }^{\bar{n}}-\widetilde{R}_{i j l}{ }^{\bar{p}} \widetilde{R}_{k \bar{p} m}{ }^{\bar{n}}-\widetilde{R}_{i j m}{ }^{p} \widetilde{R}_{k l p}{ }^{\bar{n}}-\widetilde{R}_{i j m}{ }^{\bar{p}} \widetilde{R}_{k l \bar{p}}{ }^{\bar{n}} .
\end{aligned}
$$

Case of $\alpha=\bar{i}, \beta=j, \gamma=k, \theta=l, \sigma=m, \varepsilon=\bar{n}$ in (4.8), by virtue of (4.6), we get

$$
\begin{aligned}
& ((\widetilde{R}(\widetilde{X}, \widetilde{Y}) \widetilde{R})(\widetilde{Z}, \widetilde{W}) \widetilde{U})_{\bar{i} j k l m} \bar{n} \\
& =\widetilde{R}_{\bar{i} j p}{ }^{\bar{n}} \widetilde{R}_{k l m}{ }^{p}+\widetilde{R}_{\bar{i} j \bar{p}}{ }^{\bar{n}} \widetilde{R}_{k l m}{ }^{\bar{p}}-\widetilde{R}_{\bar{i} j k}{ }^{p} \widetilde{R}_{p l m}{ }^{\bar{n}}-\widetilde{R}_{\bar{i} j k}{ }^{\bar{p}} \widetilde{R}_{\bar{p} l m}{ }^{\bar{n}} \\
& -\widetilde{R}_{\bar{i} j l}^{p} \widetilde{R}_{k p m}^{\bar{n}}-\widetilde{R}_{\bar{i} j l}{ }^{\bar{p}} \widetilde{R}_{k \bar{p} m}{ }^{\bar{n}}-\widetilde{R}_{\bar{i} j m}^{p} \widetilde{R}_{k l p}{ }^{\bar{n}}-\widetilde{R}_{\bar{i} j m}{ }^{\bar{p}} \widetilde{R}_{k l \bar{p}}{ }^{n} \\
& =R_{n p j}{ }^{i} R_{k l m}^{p}-R_{p k j}{ }^{i} R_{n m l}^{p}+R_{p l j}{ }^{i} R_{n m k}^{p}-R_{p m j}{ }^{i} R_{l k n}{ }^{p} \\
& =2 \nabla_{[n} \nabla_{m]} R_{l k j}{ }^{i}-2 \nabla_{[k} \nabla_{l]} R_{n m j}^{i} \\
& =((R(X, Y) R)(Z, W) U)_{n m l k j}^{i}-((R(X, Y) R)(Z, W) U)_{k l n m j}{ }^{k} .
\end{aligned}
$$

The other coefficients of $(\widetilde{R}(\widetilde{X}, \widetilde{Y}) \widetilde{R})(\widetilde{Z}, \widetilde{W}) \widetilde{U}$ reduce to one of (4.9), (4.10) or (4.11) by the property of the curvature tensor.

From (4.9)-(4.13), we have the following result. 
Theorem 4. Let $\nabla$ be a symmetric connection on $M$ and $T^{*} M$ be the cotangent bundle with the modified Riemannian extension $\widetilde{g}_{\nabla, c}$ over $(M, \nabla)$. Then $(\widetilde{R}(\widetilde{X}, \widetilde{Y}) \widetilde{R})(\widetilde{Z}, \widetilde{W}) \widetilde{U}=$ 0 if and only if the following conditions hold:

i) $(R(X, Y) R)(Z, W) U=0$

ii) $R_{i j k}{ }^{\bar{h}}=0$, from which it follows that $\nabla_{i} R_{h k j}{ }^{s}-\nabla_{j} R_{h k i}{ }^{s}=0$ and $\nabla_{i}\left(\nabla_{k} c_{j h}-\right.$ $\left.\nabla_{h} c_{j k}\right)-\nabla_{j}\left(\nabla_{k} c_{i h}-\nabla_{h} c_{i k}\right)-R_{i j k}{ }^{m} c_{m h}-R_{i j h}{ }^{m} c_{k m}=0$, where $R$ and $\widetilde{R}$ is the curvature tensors of the symmetric connection $\nabla$ and the Levi-Civita connection $\widetilde{\nabla}$ of the modified Riemannian extension $\widetilde{g}_{\nabla, c}$ respectively.

Theorem 4 immediately give the following result.

Corollary 1. If the symmetric connection $\nabla$ on $M$ is locally symmetric, then $(\widetilde{R}(\widetilde{X}, \widetilde{Y}) \widetilde{R})(\widetilde{Z}, \widetilde{W}) \widetilde{U}=0$ if and only if

$$
\nabla_{i}\left(\nabla_{k} c_{j h}-\nabla_{h} c_{j k}\right)-\nabla_{j}\left(\nabla_{k} c_{i h}-\nabla_{h} c_{i k}\right)-R_{i j k}{ }^{m} c_{m h}-R_{i j h}{ }^{m} c_{k m}=0 .
$$

Now, we consider the components of $\widetilde{\nabla} \widetilde{R}$. Using Proposition 1 and (4.6), by direct computation, we obtain following relations

$$
\begin{aligned}
\widetilde{\nabla}_{l} \widetilde{R}_{i j k}{ }^{h}= & \nabla_{l} R_{i j k}{ }^{h}, \\
\widetilde{\nabla}_{l} \widetilde{R}_{i j k}{ }^{h}= & p_{s}\left(\nabla_{l} \nabla_{i} R_{h k j}^{s}-\nabla_{l} \nabla_{j} R_{h k i}{ }^{s}\right)+\frac{1}{2}\left\{\nabla_{l} \nabla_{i}\left(\nabla_{k} c_{j h}-\nabla_{h} c_{j k}\right)\right. \\
& -\nabla_{l} \nabla_{j}\left(\nabla_{k} c_{i h}-\nabla_{h} c_{i k}\right)-\left(\nabla_{l} R_{i j k}{ }^{m}\right) c_{m h}-R_{i j k}{ }^{m}\left(\nabla_{l} c_{m h}\right) \\
& \left.-\left(\nabla_{l} R_{i j h}{ }^{m}\right) c_{k m}-R_{i j h}{ }^{m}\left(\nabla_{l} c_{k m}\right)\right\} \\
\widetilde{\nabla}_{l} \widetilde{R}_{i j \bar{k}}{ }^{h}= & \nabla_{l} R_{j i h}{ }^{k}, \\
\widetilde{\nabla}_{l} \widetilde{R}_{i \bar{j} k}{ }^{h}= & -\nabla_{l} R_{h k i}^{j}, \\
\widetilde{\nabla}_{l} \widetilde{R}_{\overline{i j k}}{ }^{h}= & \nabla_{l} R_{h k j}{ }^{i}, \\
\widetilde{\nabla}_{\bar{l}} \widetilde{R}_{i j k}{ }^{h}= & \nabla_{i} R_{h k j}^{l}-\nabla_{j} R_{h k i}^{l},
\end{aligned}
$$

all the others being zero, with respect to the adapted frame $\left\{E_{\alpha}\right\}$. Hence we have the following.

Theorem 5. Let $\nabla$ be a symmetric connection on $M$ and $T^{*} M$ be the cotangent bundle with the modified Riemannian extension $\widetilde{g}_{\nabla, c}$ over $(M, \nabla)$. Then $\left(T^{*} M, \widetilde{g}_{\nabla, c}\right)$ is locally symmetric if and only if $(M, \nabla)$ is locally symmetric and the components $c_{i j}$ of $c$ satisfy the condition

$$
\begin{aligned}
& \nabla_{l} \nabla_{i}\left(\nabla_{k} c_{j h}-\nabla_{h} c_{j k}\right)-\nabla_{l} \nabla_{j}\left(\nabla_{k} c_{i h}-\nabla_{h} c_{i k}\right) \\
& -R_{i j k}{ }^{m}\left(\nabla_{l} c_{m h}\right)-R_{i j h}{ }^{m}\left(\nabla_{l} c_{k m}\right) \\
= & 0 .
\end{aligned}
$$

We now turn our attention to the Ricci tensor and scalar curvature of the modified Riemannian extension $\widetilde{g}_{\nabla, c}$. Let $\widetilde{R}_{\alpha \beta}=\widetilde{R}_{\sigma \alpha \beta}{ }^{\sigma}$ and $\widetilde{r}=\left(\widetilde{g}_{\nabla, c}\right)^{\alpha \beta} \widetilde{R}_{\alpha \beta}$ denote the Ricci tensor and scalar curvature of the modified Riemannian extension $\widetilde{g}_{\nabla, c}$, respectively. From (4.6), the components of the Ricci tensor $R_{\alpha \beta}$ are characterized 
by

$$
\begin{gathered}
\widetilde{R}_{j k}=R_{j k}+\quad R_{k j} \\
\widetilde{R}_{\overline{j k}}=0, \\
\widetilde{R}_{j \bar{k}}=0, \\
\widetilde{R}_{\overline{j k}}=0,
\end{gathered}
$$

with respect to the adapted frame $\{E \alpha\}$. Next we have

Theorem 6. Let $\nabla$ be a symmetric connection on $M$ and $T^{*} M$ be the cotangent bundle with the modified Riemannian extension $\widetilde{g}_{\nabla, c}$ over $(M, \nabla)$. Then $\left(T^{*} M, \widetilde{g}_{\nabla, c}\right)$ is Ricci flat if and only if the Ricci tensor of $\nabla$ is skew symmetric (for Riemannian extension, see [17]).

Now, we operate the curvature operator to the Ricci tensor. The tensors $(\widetilde{R}(\widetilde{X}, \widetilde{Y}) \widetilde{R i c})(\widetilde{Z}, \widetilde{W})$ and $(R(X, Y) R i c)(Z, W)$ have coefficients

$$
((\widetilde{R}(\widetilde{X}, \widetilde{Y}) \widetilde{R i c})(\widetilde{Z}, \widetilde{W}))_{\alpha \beta \gamma \theta}=\widetilde{R}_{\alpha \beta \gamma} \widetilde{R}_{\varepsilon \theta}+\widetilde{R}_{\alpha \beta \theta} \widetilde{R}_{\gamma \varepsilon}
$$

and

$$
((R(X, Y) R i c)(Z, W))_{i j k l}=R_{i j k}^{p} R_{p l}+R_{i j l}^{p} R_{k p}
$$

respectively. By putting $\alpha=i, \beta=j, \gamma=k, \theta=l$, it follows that

$$
\begin{aligned}
((\widetilde{R}(\widetilde{X}, \widetilde{Y}) \widetilde{R i c})(\widetilde{Z}, \widetilde{W}))_{i j k l} & =\widetilde{R}_{i j k}^{p} \widetilde{R}_{p l}+\widetilde{R}_{i j l}^{p} \widetilde{R}_{k p} \\
& =R_{i j k}{ }^{p}\left(R_{p l}+R_{l p}\right)+R_{i j l}^{p}\left(R_{k p}+R_{p k}\right)
\end{aligned}
$$

all the others being zero. Let the base manifold $M$ be a Riemannian manifold with the metric $g$ and $\nabla$ be the Levi-Civita connection of $g$. Then

$$
\begin{aligned}
((\widetilde{R}(\widetilde{X}, \widetilde{Y}) \widetilde{R i c})(\widetilde{Z}, \widetilde{W}))_{i j k l} & =2 R_{i j k}^{p} R_{p l}+2 R_{i j l}^{p} R_{k p} \\
& =2((R(X, Y) R i c)(Z, W))_{i j k l} .
\end{aligned}
$$

Therefore we get the following.

Theorem 7. Let $\nabla$ be the Levi-Civita connection on the Riemannian manifold $(M, g)$ and $T^{*} M$ be its cotangent bundle with the modified Riemannian extension $\widetilde{g}_{\nabla, c}$. Then $(\widetilde{R}(\widetilde{X}, \widetilde{Y}) \widetilde{R i c})(\widetilde{Z}, \widetilde{W})=0$ if and only if $(R(X, Y) R i c)(Z, W)=0$.

From (4.2) and (4.16), the scalar curvature of the modified Riemannian extension $\widetilde{g}_{\nabla, c}$ is given by

$$
\widetilde{r}=0 .
$$

Theorem 8. Let $\nabla$ be a symmetric connection on $M$ and $T^{*} M$ be the cotangent bundle with the modified Riemannian extension $\widetilde{g}_{\nabla, c}$ over $(M, \nabla)$. Then $\left(T^{*} M, \widetilde{g}_{\nabla, c}\right)$ is a space of constant scalar curvature 0 .

In the following we give the conditions under which the cotangent bundle $\left(T^{*} M, \widetilde{g}_{\nabla, c}\right)$ is locally conformally flat. The cotangent bundle $T^{*} M$ with the modified Riemannian extension $\widetilde{g}_{\nabla, c}$ is locally conformally flat if and only if its Weyl tensor $\widetilde{W}$ vanishes, where the Weyl tensor is given by

$$
\begin{aligned}
\widetilde{W}_{\alpha \beta \gamma \sigma}= & \widetilde{R}_{\alpha \beta \gamma \sigma}+\frac{\widetilde{r}}{2(2 n-1)(n-1)}\left\{\left(\widetilde{g}_{\nabla, c}\right)_{\alpha \gamma}\left(\widetilde{g}_{\nabla, c}\right)_{\beta \sigma}-\left(\widetilde{g}_{\nabla, c}\right)_{\alpha \sigma}\left(\widetilde{g}_{\nabla, c}\right)_{\beta \gamma}\right\} \\
& -\frac{1}{2(n-1)}\left(\left(\widetilde{g}_{\nabla, c}\right)_{\beta \sigma} \widetilde{R}_{\alpha \gamma}-\left(\widetilde{g}_{\nabla, c}\right)_{\alpha \sigma} \widetilde{R}_{\beta \gamma}+\left(\widetilde{g}_{\nabla, c}\right)_{\alpha \gamma} \widetilde{R}_{\beta \sigma}-\left(\widetilde{g}_{\nabla, c}\right)_{\beta \gamma} \widetilde{R}_{\alpha \sigma}\right),
\end{aligned}
$$


where $\widetilde{R}_{\alpha \beta \gamma \sigma}=\widetilde{R}_{\alpha \beta \gamma}{ }^{\lambda}\left(\widetilde{g}_{\nabla, c}\right)_{\lambda \sigma}$.

From 4.6, we obtain

$$
\begin{gathered}
\widetilde{R}_{i j k n}=R_{i j k}^{h} c_{h n}+p_{s}\left(\nabla_{i} R_{n k j}{ }^{s}-\nabla_{j} R_{n k i}{ }^{s}\right) \\
+\frac{1}{2}\left\{\nabla_{i}\left(\nabla_{k} c_{j n}-\nabla_{n} c_{j k}\right)-\nabla_{j}\left(\nabla_{k} c_{i n}-\nabla_{n} c_{i k}\right)-R_{i j k}{ }^{h} c_{h n}-R_{i j n}{ }^{h} c_{k h}\right\} \\
\widetilde{R}_{i j k \bar{n}}=R_{i j k}{ }^{n} \\
\widetilde{R}_{i j \bar{k} n}=R_{j i n}{ }^{k} \\
\widetilde{R}_{i \bar{j} k n}=R_{k n i}{ }^{j} \\
\widetilde{R}_{\bar{i} j k n}=R_{n k j}{ }^{i} \\
\text { otherwise }=0 .
\end{gathered}
$$

The non-zero components of Weyl tensor of the modified Riemannian extension $\widetilde{g}_{\nabla, c}$ are given by

$$
\begin{aligned}
\widetilde{W}_{i j k n}= & \widetilde{R}_{i j k n}-\frac{1}{2(n-1)}\left\{c_{j n}\left(R_{i k}+R_{k i}\right)-c_{i n}\left(R_{j k}+R_{k j}\right)\right. \\
& \left.+c_{i k}\left(R_{j n}+R_{n j}\right)-c_{j k}\left(R_{i n}+R_{n i}\right)\right\} \\
\widetilde{W}_{i j k \bar{n}}= & \widetilde{R}_{i j k \bar{n}}-\frac{1}{2(n-1)}\left(\delta_{j}^{n}\left(R_{i k}+R_{k i}\right)-\delta_{i}^{n}\left(R_{j k}+R_{k j}\right)\right) \\
\widetilde{W}_{i j \bar{k} n}= & \widetilde{R}_{i j \bar{k} n}-\frac{1}{2(n-1)}\left(\delta_{i}^{k}\left(R_{j n}+R_{n j}\right)-\delta_{j}^{k}\left(R_{i n}+R_{n i}\right)\right) \\
\widetilde{W}_{i \bar{j} k n}= & \widetilde{R}_{i \bar{j} k n}-\frac{1}{2(n-1)}\left(\delta_{n}^{j}\left(R_{i k}+R_{k i}\right)-\delta_{j}^{k}\left(R_{i n}+R_{n i}\right)\right) \\
\widetilde{W}_{\bar{i} j k n}= & \widetilde{R}_{\bar{i} j k n}-\frac{1}{2(n-1)}\left(\delta_{k}^{i}\left(R_{j n}+R_{n j}\right)-\delta_{n}^{i}\left(R_{j k}+R_{k j}\right)\right)
\end{aligned}
$$

Using the same way in 2, we can say the same result in 2] by means of adapted frame.

Theorem 9. Let $\nabla$ be a symmetric connection on $M$ and $T^{*} M$ be the cotangent bundle with the modified Riemannian extension $\widetilde{g}_{\nabla, c}$ over $(M, \nabla)$. Then $\left(T^{*} M, \widetilde{g}_{\nabla, c}\right)$ is locally conformally flat if and only if $(M, \nabla)$ is projectively flat and the components $c_{i j}$ of $c$ satisfy the condition

$\nabla_{i}\left(\nabla_{k} c_{j n}-\nabla_{n} c_{j k}\right)-\nabla_{j}\left(\nabla_{k} c_{i n}-\nabla_{n} c_{i k}\right)-R_{i j k}{ }^{h} c_{h n}-R_{i j n}{ }^{h} c_{k h}=0$ (also see, [2]).

Finally, we finish the section by the projective curvature tensor of the modified Riemannian extension $\widetilde{g}_{\nabla, c}$. A manifold is said to be projectively flat if the projective curvature tensor vanishes. The projective curvature tensor is defined by

$$
\widetilde{P}_{\alpha \beta \gamma \sigma}=\widetilde{R}_{\alpha \beta \gamma \sigma}-\frac{1}{(2 n-1)}\left(\left(\widetilde{g}_{\nabla, c}\right)_{\alpha \sigma} \widetilde{R}_{\beta \gamma}-\left(\widetilde{g}_{\nabla, c}\right)_{\beta \sigma} \widetilde{R}_{\alpha \gamma}\right) .
$$

Case of $\alpha=i, \beta=j, \gamma=\bar{k}, \sigma=n$, we have

$$
\begin{aligned}
\widetilde{P}_{i j \bar{k} n} & =\widetilde{R}_{i j \bar{k} n}-\frac{1}{(2 n-1)}\left(\left(\widetilde{g}_{\nabla, c}\right)_{i n} \widetilde{R}_{j \bar{k}}-\left(\widetilde{g}_{\nabla, c}\right)_{j n} \widetilde{R}_{i \bar{k}}\right) \\
& =R_{j i n}{ }^{k} .
\end{aligned}
$$


Case of $\alpha=i, \beta=j, \gamma=k, \sigma=n$, we obtain

$$
\begin{aligned}
\widetilde{P}_{i j k n}= & \widetilde{R}_{i j k n}-\frac{1}{(2 n-1)}\left(\left(\widetilde{g}_{\nabla, c}\right)_{i n} \widetilde{R}_{j k}-\left(\widetilde{g}_{\nabla, c}\right)_{j n} \widetilde{R}_{i k}\right) \\
= & R_{i j k}^{h} c_{h n}+p_{s}\left(\nabla_{i} R_{n k j}^{s}-\nabla_{j} R_{n k i}^{s}\right) \\
& +\frac{1}{2}\left\{\nabla_{i}\left(\nabla_{k} c_{j n}-\nabla_{n} c_{j k}\right)-\nabla_{j}\left(\nabla_{k} c_{i n}-\nabla_{n} c_{i k}\right)-R_{i j k}^{h} c_{h n}-R_{i j n}^{h} c_{k h}\right\} \\
& -\frac{1}{(2 n-1)}\left(c_{i n}\left(R_{j k}+R_{k j}\right)-c_{j n}\left(R_{i k}+R_{k i}\right)\right) .
\end{aligned}
$$

The above equations give the following result.

Theorem 10. Let $\nabla$ be a symmetric connection on $M$ and $T^{*} M$ be the cotangent bundle with the modified Riemannian extension $\widetilde{g}_{\nabla, c}$ over $(M, \nabla)$. Then $\left(T^{*} M, \widetilde{g}_{\nabla, c}\right)$ is projectively flat if and only if $(M, \nabla)$ is flat and the components $c_{i j}$ of $c$ satisfy the condition

$$
\nabla_{i}\left(\nabla_{k} c_{j n}-\nabla_{n} c_{j k}\right)-\nabla_{j}\left(\nabla_{k} c_{i n}-\nabla_{n} c_{i k}\right)=0 .
$$

Remark 1. i) If $c_{i j}=0$, then the conditions 4.7, 4.14, 4.15, 4.17) and 4.18) are identically fulfilled.

ii) If $c_{i j}$ is parallel with respect to $\nabla$, then the conditions (4.7), (4.14), 4.15), 4.17) and (4.18) are identically fulfilled.

iii) If $c_{i j}$ satisfies the relation $\nabla_{i} c_{j k}-\nabla_{j} c_{i k}=\nabla_{k} \omega_{i j}$, where the components $\omega_{i j}$ define a 2 -form on $M$ and if $(M, \nabla)$ is flat then the condition (4.7), 4.14), 4.15), (4.17) and (4.18) are identically verified.

\section{Curvature properties of Another metric Connection of the MODIFIED RIEMANNIAN EXTENSION $\widetilde{g}_{\nabla, c}$}

Let $\nabla$ be a linear connection on an $n$-dimensional differentiable manifold $M$. The connection $\nabla$ is symmetric if its torsion tensor vanishes, otherwise it is nonsymmetric. If there is a Riemannian metric $g$ on $M$ such that $\nabla g=0$, then the connection $\nabla$ is a metric connection, otherwise it is non-metric. It is well known that a linear connection is symmetric and metric if and only if it is the Levi-Civita connection. In section 4 , we have considered the Levi-Civita connection $\widetilde{\nabla}$ of the modified Riemannian extension $\widetilde{g}_{\nabla, c}$ on the cotangent bundle $T^{*} M$ over $(M, \nabla)$. The connection is the unique connection which satisfies $\widetilde{\nabla}_{\alpha}\left(\widetilde{g}_{\nabla, c}\right)_{\beta \gamma}=0$ and has a zero torsion. Hayden [9] introduced a metric connection with a non-zero torsion on a Riemannian manifold. Now we are interested in a metric connection ${ }^{(M)} \widetilde{\nabla}$ of the modified Riemannian extension $\widetilde{g}_{\nabla, c}$ whose torsion tensor ${ }^{(M)} \widetilde{\nabla}_{T_{\gamma \beta}^{\varepsilon}}$ is skewsymmetric in the indices $\gamma$ and $\beta$. We denote components of the connection ${ }^{(M)} \widetilde{\nabla}$ by ${ }^{(M)} \widetilde{\Gamma}$. The metric connection ${ }^{(M)} \widetilde{\nabla}$ satisfies

$$
{ }^{(M)} \widetilde{\nabla}_{\alpha}\left(\widetilde{g}_{\nabla, c}\right)_{\beta \gamma}=0 \text { and }{ }^{(M)} \widetilde{\Gamma}_{\alpha \beta}^{\gamma}-{ }^{(M)} \widetilde{\Gamma}_{\beta \alpha}^{\gamma}={ }^{(M)} \widetilde{\nabla}_{T \beta}^{\gamma} .
$$

On the equation (5.1) is solved with respect to ${ }^{(M)} \widetilde{\Gamma}_{\alpha \beta}^{\gamma}$, one finds the following solution 9.

$$
{ }^{(M)} \widetilde{\Gamma}_{\alpha \beta}^{\gamma}=\widetilde{\Gamma}_{\alpha \beta}^{\gamma}+\widetilde{U}_{\alpha \beta}^{\gamma},
$$


where $\widetilde{\Gamma}_{\alpha \beta}^{\gamma}$ is components of the Levi-Civita connection of the modified Riemannian extension $\widetilde{g}_{\nabla, c}$,

$$
\widetilde{U}_{\alpha \beta \gamma}=\frac{1}{2}\left({ }^{(M)} \widetilde{\nabla} T_{\alpha \beta \gamma}+{ }^{(M)} \widetilde{\nabla} T_{\gamma \alpha \beta}+{ }^{(M)} \widetilde{\nabla} T_{\gamma \beta \alpha}\right)
$$

and

$$
\widetilde{U}_{\alpha \beta \gamma}=\widetilde{U}_{\alpha \beta}^{\epsilon}\left(\widetilde{g}_{\nabla, c}\right)_{\epsilon \gamma},{ }^{(M)} \widetilde{\nabla}_{T \beta \gamma}=T_{\alpha \beta}^{\epsilon}\left(\widetilde{g}_{\nabla, c}\right)_{\epsilon \gamma}
$$

If we put

$$
{ }^{\left({ }^{M}\right)} \widetilde{\nabla} T_{i j}^{\bar{r}}=-p_{s} R_{i j r}{ }^{s}
$$

all other ${ }^{\left({ }^{M}\right)} \widetilde{\nabla} T_{\alpha \beta}^{\gamma}$ not related to ${ }^{(M)} \widetilde{\nabla} T_{i j}^{\bar{r}}$ being assumed to be zero. We choose this ${ }^{(M)} \widetilde{\nabla} T_{\alpha \beta}^{\gamma}$ in $T^{*} M$ which is skew-symmetric in the indices $\gamma$ and $\beta$ as torsion tensor and determine a metric connection in $T^{*} M$ with respect to the modified Riemannian extension $\widetilde{g}_{\nabla, c}$. By using (5.3) and (5.4), we get non-zero component of $\widetilde{U}_{\alpha \beta}^{\gamma}$ as follows:

$$
\widetilde{U}_{i j}^{\bar{h}}=p_{s} R_{j h i}^{s}
$$

with respect to the adapted frame. From (5.2) and Proposition 1, we have

Proposition 3. Let $\nabla$ be a symmetric connection on $M$ and $T^{*} M$ be the cotangent bundle with the modified Riemannian extension $\widetilde{g}_{\nabla, c}$ over $(M, \nabla)$. The metric connection ${ }^{(M)} \widetilde{\nabla}$ with respect to $\widetilde{g}_{\nabla, c}$ is as follows:

$$
\begin{aligned}
& { }^{(M)} \widetilde{\nabla}_{E_{\bar{i}}} E_{\bar{j}}=0,{ }^{(M)} \widetilde{\nabla}_{E_{\bar{i}}} E_{j}=0, \\
& { }^{(M)} \widetilde{\nabla}_{E_{i}} E_{\bar{j}}=-\Gamma_{i h}^{j} E_{\bar{h}}, \\
& { }_{(M)} \widetilde{\nabla}_{E_{i}} E_{j}=\Gamma_{i j}^{h} E_{h}+\frac{1}{2}\left(\nabla_{i} c_{j h}+\nabla_{j} c_{i h}-\nabla_{h} c_{i j}\right) E_{\bar{h}}
\end{aligned}
$$

with respect to the adapted frame, where $R_{h j i}{ }^{s}$ are the local coordinate components of the curvature tensor field $R$ of the symmetric connection $\nabla$ on $M$.

Remark 2. If $\nabla_{i} c_{j h}+\nabla_{j} c_{i h}-\nabla_{h} c_{i j}=0$, the metric connection ${ }^{(M)} \widetilde{\nabla}$ in $T^{*} M$ of the modified Riemannian extension $\widetilde{g}_{\nabla, c}$ coincides with the metric connection ${ }^{H} \nabla$ of the Riemannian extension $\widetilde{g}_{\nabla}$, where ${ }^{H} \nabla$ is the horizontal lift of the symmetric connection $\nabla$ on $M$.

For the curvature tensor ${ }^{(M)} \widetilde{R}$ of the metric connection ${ }^{(M)} \widetilde{\nabla}$, we state the following result.

Proposition 4. Let $\nabla$ be a symmetric connection on $M$ and $T^{*} M$ be the cotangent bundle with the modified Riemann extension $\widetilde{g}_{\nabla, c}$ over $(M, \nabla)$. The curvature tensor ${ }^{(M)} \widetilde{R}$ of the metric connection ${ }^{(M)} \widetilde{\nabla}$ satisfies the followings:

$$
\begin{aligned}
{ }^{(M)} \widetilde{R}\left(E_{i}, E_{j}\right) E_{k}= & R_{i j k}{ }^{h} E_{h} \\
& +\frac{1}{2}\left\{\nabla_{i}\left(\nabla_{k} c_{j h}-\nabla_{h} c_{j k}\right)-\nabla_{j}\left(\nabla_{k} c_{i h}-\nabla_{h} c_{i k}\right)\right. \\
& \left.\left.-R_{i j k}{ }^{m} c_{m h}-R_{i j h}{ }^{m} c_{k m}\right\}\right\} E_{\bar{h}} \\
{ }^{(M)} \widetilde{R}\left(E_{i}, E_{j}\right) E_{\bar{k}}= & R_{j i h}{ }^{k} E_{\bar{h}} \\
{ }_{(M)} \widetilde{R}\left(E_{i}, E_{\bar{j}}\right) E_{k}= & 0,{ }^{(M)} \widetilde{R}\left(E_{i}, E_{\bar{j}}\right) E_{\bar{k}}=0,{ }^{(M)} \widetilde{R}\left(E_{\bar{i}}, E_{j}\right) E_{k}=0 \\
{ }^{(M)} \widetilde{R}\left(E_{\bar{i}}, E_{j}\right) E_{\bar{k}}= & 0,{ }^{(M)} \widetilde{R}\left(E_{\bar{i}}, E_{\bar{j}}\right) E_{k}=0,{ }^{(M)} \widetilde{R}\left(E_{\bar{i}}, E_{\bar{j}}\right) E_{\bar{k}}=0
\end{aligned}
$$


with respect to the adapted frame.

The non-zero component of the contracted curvature tensor field (Ricci tensor field) ${ }^{(M)} \widetilde{R}_{\gamma \beta}={ }^{(M)} \widetilde{R}_{\alpha \beta \gamma}{ }^{\alpha}$ of the metric connection ${ }^{(M)} \widetilde{\nabla}$ is as follows:

$$
{ }^{(M)} \widetilde{R}_{j k}=R_{j k} .
$$

For the scalar curvature ${ }^{(M)} \widetilde{r}$ of the metric connection ${ }^{(M)} \widetilde{\nabla}$ with respect to $\widetilde{g}_{\nabla, c}$, we obtain

$$
{ }^{(M)} \widetilde{r}=0 .
$$

Thus we have the following theorem.

Theorem 11. The cotangent bundle $T^{*} M$ with the metric connection ${ }^{(M)} \widetilde{\nabla}$ has a vanishing scalar curvature with respect to the modified Riemannian extension $\widetilde{g}_{\nabla, c}$.

\section{REFERENCES}

[1] S. Aslanci, S. Kazimova, A. A. Salimov, Some remarks concerning Riemannian extensions. Ukrainian Math. J. 62 (2010), no. 5, 661-675.

[2] Z. Afifi, Riemann extensions of affine connected spaces. Quart. J. Math., Oxford Ser. (2) 5(1954), 312-320.

[3] E. Calvino-Louzao, E. García-Río, P. Gilkey and A. Vazquez-Lorenzo, The geometry of modified Riemannian extensions. Proc. R. Soc. Lond. Ser. A Math. Phys. Eng. Sci. 465(2009), no. 2107, 2023-2040.

[4] E. Calviño-Louzao, E. García-Río and R. Vázquez-Lorenzo, Riemann extensions of torsionfree connections with degenerate Ricci tensor. Can. J. Math. 62(2010), No. 5, 1037-1057.

[5] A. Derdzinski, Connections with skew-symmetric Ricci tensor on surfaces. Results Math. 52(2008), no. 3-4, 223-245.

[6] L. S. Druţă, Classes of general natural almost anti-Hermitian structures on the cotangent bundles. Mediterr. J. Math. 8 (2011), no. 2, 161-179.

[7] V. Dryuma, The Riemann extensions in theory of differential equations and their applications. Mat.Fiz. Anal. Geom. 10(2003), no. 3, 307-325.

[8] E. Garcia-Rio, D. N. Kupeli, M. E. Vazquez-Abal, and R. Vazquez-Lorenzo, Affine Osserman connections and their Riemann extensions. Differential Geom. Appl. 11(1999), no. 2, 145-153.

[9] H. A. Hayden, Sub-spaces of a space with torsion. Proc. London Math. Soc. S2-34 (1932), $27-50$.

[10] T. Ikawa, K. Honda, On Riemann extension. Tensor (N.S.) 60 (1998), no. 2, 208-212.

[11] M. Iscan, A. A. Salimov, On Kähler-Norden manifolds. Proc. Indian Acad. Sci. Math. Sci. 119 (2009), no. 1, 71-80.

[12] O. Kowalski and M. Sekizawa, On natural Riemann extensions. Publ. Math. Debrecen 78(2011), no. 3-4, 709-721.

[13] G. I. Kruchkovich, Hypercomplex structure on manifold. I, Tr. Sem. Vect. Tens. Anal, Moscow Univ., 16 (1972), 174-201.

[14] K. P. Mok, Metrics and connections on the cotangent bundle. Kodai Math. Sem. Rep. 28 (1976/77), no. 2-3, 226-238.

[15] V. Oproiu, N. Papaghiuc, On the cotangent bundle of a differentiable manifold. Publ. Math. Debrecen 50 (1997), no. 3-4, 317-338.

[16] V. Oproiu, N. Papaghiuc, Some examples of almost complex manifolds with Norden metric. Publ. Math. Debrecen 41 (1992), no. 3-4, 199-211.

[17] E. M. Patterson and A. G.Walker, Riemann extensions. Quart. J. Math. Oxford Ser. (2) 3(1952), 19-28.

[18] M. Sekizawa, Natural transformations of affine connections on manifolds to metrics on cotangent bundles. Proceedings of the 14th winter school on abstract analysis (Srni, 1986), Rend. Circ. Mat. Palermo (2) Suppl. No. 14(1987), 129-142.

[19] M. Toomanian, Riemann extensions and complete lifts of s-spaces. Ph. D. Thesis, The university, Southampton, 1975.

[20] S. Tachibana, Analytic tensor and its generalization. Tohoku Math. J., 12 (1960), no.2, 208221 
[21] L. Vanhecke and T. J. Willmore, Riemann extensions of D'Atri spaces. Tensor (N.S.) 38(1982), 154-158.

[22] T. J.Willmore, Riemann extensions and affine differential geometry. Results Math. 13(1988), no. 3-4, 403-408.

[23] K. Yano, M. Ako, On certain operators associated with tensor field. Kodai Math. Sem. Rep., 20 (1968), 414-436.

[24] K. Yano, S. Ishihara, Tangent and Cotangent Bundles. Marcel Dekker, Inc., New York 1973.

Ataturk University, Faculty of Science, Department of Mathematics, 25240, ErzurumTURKEY.

E-mail address: agezer@atauni.edu.tr

Igdir University, Igdir Vocational School, 76000, Igdir-Turkey.

E-mail address: lokman.bilen@igdir.edu.tr

Ataturk University, Faculty of Science, Department of Mathematics, 25240, ErzurumTURKEY.

E-mail address: ali.cakmak@atauni.edu.tr 